\title{
$\$$ Research Square \\ Deformation Mediated by Grain Rotation in Superhard Nanocrystalline cBN
}

Pei Wang ( $\nabla$ peiwang1108@gmail.com )

Southern University of Science and Technology

\section{Kim Kisslinger}

Brookhaven National Laboratory, Center for Functional Nanomaterials

\section{Haiyan Chen}

New Jersey Institute of Technology

\section{Yusheng Zhao}

Southern University of Science \& Technology

\section{Liping Wang}

Southern University of Science and Technology https://orcid.org/0000-0002-6137-3113

\section{Article}

Keywords: lattice strain analysis, deformation

Posted Date: December 28th, 2020

DOI: https://doi.org/10.21203/rs.3.rs-124087/v1

License: (c) (1) This work is licensed under a Creative Commons Attribution 4.0 International License. Read Full License 


\title{
Deformation Mediated by Grain Rotation in Superhard Nanocrystalline cBN
}

\author{
Pei Wang ${ }^{1,2^{*}}$, Kim Kisslinger ${ }^{3}$, Haiyan $\mathrm{Chen}^{4}$, Yusheng Zhao ${ }^{1,2^{*}}$ and Liping Wang ${ }^{1,2^{*}}$ \\ ${ }^{1}$ Academy for Advanced Interdisciplinary Studies, and Shenzhen Engineering Research Center \\ for Frontier Materials Synthesis at High Pressures, Southern University of Science and \\ Technology (SUSTech), Shenzhen 518055, P. R. China \\ ${ }^{2}$ Department of Physics, Southern University of Science and Technology (SUSTech), Shenzhen, \\ 518055, P. R. China \\ ${ }^{3}$ Center for Functional Nanomaterials, Brookhaven National Laboratory, Upton, New York 11973, \\ USA \\ ${ }^{4}$ Mineral Physics Institute, Stony Brook University, Stony Brook, New York 11794, USA
}

\begin{abstract}
Superhard materials such as diamond and cubic boron nitride (cBN) are becoming ever more scientifically and technologically important, and critical and fundamental knowledge about their constitutive properties and deformational mechanisms is in increasingly high demand. Although it has long been suggested by theoretical modeling that deformation of face-centered cubic superhard materials is dominated by Shockley partial dislocations and screw dislocations, there has been a glaring lack of experimental evidence. Here, we report in situ deformation experiments of nanocrystalline $\mathrm{cBN}$ (nc-cBN) samples at high pressures and

\footnotetext{
* Corresponding authors: peiwang1108@gmail.com, zhaoys@ @sustech.edu.cn \& wanglp3@ sustech.edu.cn
} 
temperatures using a deformation-DIA (D-DIA) apparatus coupled with synchrotron $\mathrm{X}$-ray diffraction techniques. Intrinsic stress-strain relations have been obtained for nc-cBN for the first time, and only elastic deformation occurred up to a strain of at least $14 \%$ at room temperature (RT), demonstrating its remarkable strength, which was undoubtedly enhanced by observed microscopic features such as the LomerCottrell (L-C) locks and low-angle GBs. While deformation at RT is dominated by brittle fractures and mechanic crushing induced by grain boundary twisting mediated by full dislocations, plasticity of nc-cBN at higher temperatures is controlled by grain rotation and twinning mediated by Shockley partial dislocations. At 4.0 GPa and $1200{ }^{\circ} \mathrm{C}$, accumulated shear strain resulted in the conversion of $\mathrm{cBN}$ to $\mathrm{hBN}$ at or near twisting GBs, releasing stress and mediating deformation in the process. We demonstrate the apparent agreement between the differential micro-stress derived from peak broadening analysis and differential macro-stress deduced from lattice strain analysis. 


\section{Introduction}

Cubic boron nitride (cBN) as conventional superhard material is technologically significant because of its excellent physical and chemical properties ${ }^{1-9}$. It is widely used for cutting, grinding, drilling and coating under some of the harshest environments. Superior chemical inertness and thermal stability $\left(\sim 1380{ }^{\circ} \mathrm{C}\right)^{10}$ make cBN the only choice in place of diamond for high-speed cutting of hardened steel ${ }^{3}$. Furthermore, $\mathrm{cBN}$ is a promising candidate for use in the extreme conditions associated with hypersonic flight, scramjet propulsion, atmospheric reentry, and rocket propulsion ${ }^{11}$ due to its high melting temperature $\left(>3000{ }^{\circ} \mathrm{C}\right)^{12}$, high thermal conductivity $\left(740 \mathrm{~W} \cdot \mathrm{K}^{-1}\right)^{13}$, high thermal shock resistance, low thermal expansion coefficient $\left(1.2 \times 10^{-6} /{ }^{\circ} \mathrm{C}\right)^{14}$, and excellent chemical inertness and mechanical strength (Vickers hardness of single crystal cBN: $\sim 50 \mathrm{GPa})^{1-5,9,15}$. These potential applications demand a better knowledge of deformational and constitutive properties of $\mathrm{cBN}$ under extreme environment. However, as the second hardest material known to mankind, $\mathrm{cBN}$ is experimentally difficult to be deformed, particularly at room temperature (RT). Current knowledge of plastic deformation in $\mathrm{cBN}$ or other superhard materials is mainly based on theoretical simulations and experiments under uncontrolled stress conditions $^{16-21}$. Traditionally, the strength of superhard material can be estimated using bending, indentation and grinding at ambient pressure ${ }^{20,21}$ or via high-pressure experiments under nonhydrostatic compression ${ }^{22-26}$, but resultant compressional or tensile strength from these studies are generally inconsistent. In addition, the strength can also be derived from analyzing diffraction peak broadening during hydrostatic 
compression (i.e., no external stress field) of polycrystalline samples under highpressure and high temperature ${ }^{27-30}$. Because previous experimental methodologies could not define the stress applied during deformation, the intrinsic strain-stress relation, which is fundamentally important to understanding the elasticity and plasticity of $\mathrm{cBN}$, has yet to be established under both room and elevated temperatures.

The mechanical performance of materials is controlled by their intrinsic deformation mechanisms and dominant dislocation structures ${ }^{31-37}$. In-depth understanding of such microscopic processes or features is therefore imperative to improving effectively the mechanical properties of materials. Nanocrystalline (nc) materials have been demonstrated to be comparatively stronger than their coarsegrained counterparts via work hardening. For example, nanotwinned $\mathrm{cBN}$ and diamond prepared at ultrahigh pressure and temperature have been shown to be twice as hard and several times as tough as coarse-grained $\mathrm{cBN}$ and diamond, respectively ${ }^{1,}$ ${ }^{38}$. A slew of mechanisms including twinning, partial and screw dislocations emitting from grain boundaries (GBs), grain boundary sliding, dislocation slip, grain rotation and even superstructures have been proposed for the deformation of superhard nc materials $^{33,37,39,40}$. Conventional Read-Shockley model speculates that the partial dislocations at twin boundaries or a network of screw dislocations at a twist boundary govern the deformational behavior of materials with face-centered cubic (fcc) diamond structure ${ }^{41-44}$. However, the dominant deformation mechanism has been debated extensively in the theoretical literature due to lack of sufficient experimental 
evidence. Moreover, because a deformational screw dislocation is usually accompanied by large shear stress and mixed with local partial dislocations, interactions between screw dislocation and edge dislocations at grain boundaries ineluctably take place and even induce the formation of superstructure, phase transition and new transformation paths to relax shear-dominated stresses at concentrators ${ }^{45,46}$. Understanding the effects of these interactions and the evolution of dislocations is conducive to designing nc materials with superior mechanical and physical properties.

To address the aforementioned questions, we have systematically investigated the intrinsic strain-stress relations and evolution of deformation mechanisms of nc$\mathrm{cBN}$ at high pressures via in situ deformation experiments in a deformation-DIA (DDIA) apparatus ${ }^{47-52}$ and microscopic studies on the recovered samples using transmission electron microscopy (TEM). The main dislocation mode at RT is manifested as GB twisting mediated by full dislocations on (111) planes for nc-cBN without plastic yielding at uniaxial strains up to $14 \%$. Cubic BN crystals showed remarkable ductile flow and plastic deformation at $1000{ }^{\circ} \mathrm{C}$ through GB twisting mediated by Shockley partial dislocations. Surprisingly, high shear stress even induced the formation of superstructures and local phase transition at higher pressure and temperature $\left(4.0 \mathrm{GPa} / 1200{ }^{\circ} \mathrm{C}\right)$.

\section{Results and Discussion}

Experimental setups and procedures Shown in Figure 1a is a schematic diagram illustrating the setups for uniaxial deformation experiments at high pressure 
and temperature using a D-DIA-type cubic anvil apparatus coupled with synchrotron $\mathrm{X}$-radiation. Energy-dispersive XRD data were collected with a counting-time of $\sim 10$ minutes using a ten-element Ge solid-state detector (SSD) on which all elements are arranged in a circle and each element is located at a fixed azimuth angle $\psi\left(22.5^{\circ}\right.$ apart from $0^{\circ}-180^{\circ}$, plus one at $270^{\circ}$ ). To eliminate unwanted diffraction signals from materials surrounding the sample, a conical-slit system located between the sample and the detectors was employed to collimate and constrain the $2 \theta$ angle of the diffracted x-rays. Radiographic images were also taken, with data collection toggling continuously between diffraction and imaging modes. An amorphous boron-epoxy cube was used as pressure medium, as shown in Fig. 1b. Dense $\mathrm{Al}_{2} \mathrm{O}_{3}$ pistons were placed at both ends of specimens, which were pre-compressed into pellets and packed into a hexagonal boron nitride (hBN) capsule; three $25-\mu \mathrm{m}$-thick tantalum disks were placed at the piston-specimen and specimen-specimen contacts as strain markers. The nc-cBN sample was first compressed to desired pressure at RT and then annealed at high temperature $\left(\sim 1000{ }^{\circ} \mathrm{C}\right)$ for 1 hour using enclosed resistive graphite heater. Figure 1c shows the (111) diffraction line of nc-cBN along the entire compression, annealing, and deformation path. Figures S1a and b show all diffraction peaks of nc$\mathrm{cBN}$ at azimuth angles of $180^{\circ}$ (axial direction) and $67.5^{\circ}$ at various experimental stages and conditions. As the detector elements at the azimuth angles of $90^{\circ}$ and $270^{\circ}$ (radial direction) weren't available during all experiments, signals recorded by the element situated at $67.5^{\circ}$ are taken as the proxies for those from the radial direction in this study. Furthermore, because diffraction peaks (200), (220) and (311) were either 
very weak or even absent during high-pressure deformational experiments (Fig. S1), the stress analysis in this study is based on the diffraction peak (111) only.

Stress-strain measurements and analyses Total axial strains $\left(\varepsilon_{t}\right)$ due to deformation under high pressure and temperature conditions are determined by measuring the length of the specimen defined by the strain markers (i.e., Ta foils) from the radiographic images according to the following formula: $\varepsilon_{t}=\left(l_{0}-l_{t}\right) / l_{0}$, where $l_{t}$ is the sample length at given stress and $l_{0}$ the sample length at the onset of deformation. Microscopic deviatoric stress generated at grain contacts contributes to diffraction peak broadening. As the microscopic strain $\left(\varepsilon_{m}\right)$ revealing the distribution of longitudinal strain parallel to the diffraction vector is the amount of line broadening $^{27-30,48}$, it can be calculated from the full width at half maximum (FWHM) of various diffraction lines based on the following formula:

$$
\varepsilon_{m}=\frac{\sqrt{W_{\text {obs. }}{ }^{2}-W_{\text {ins. }}{ }^{2}-W_{\text {size }}{ }^{2}}}{E(P, T)}
$$

where $W_{\text {obs. }}, W_{\text {ins. }}$ and $W_{\text {size }}$ are the observed diffraction line width of sample during deformation, the peak width of stress-free sample and the peak broadening contributions from the crystal size, respectively, and $E(P, T)$ the x-ray photon energy of corresponding lattice plane. In consideration of average grain size $(\sim 37 \mathrm{~nm})$ of the raw nc-cBN samples, we estimated the microstrain contributed by grain size based on the Scherrer formula:

$$
L=\frac{k h c}{W_{\operatorname{size}(2 \theta)} E \cos \theta}
$$

where $L$ is the grain size, $k$ the Scherrer constant correlated to crystallite shape, 
normally taken as $0.9, h$ the Planck constant, and $W_{\text {size }(2 \theta)}$ the FWHM at a fixed wavelength $\lambda$, in angular-dispersive mode. To calculate the FWHM $\left(W_{\operatorname{size}(E)}\right)$ at a given x-ray photon energy $E$ in energy-dispersive mode, the Scherrer formula can be rewritten as follows via differentiating Bragg's diffraction equation, $h c=2 E d \sin \theta$, with respect to $E$ and $2 \theta$, respectively:

$$
W_{\text {size }(E)}=\frac{k h c}{2 L \sin \theta}
$$

where grain size $L$ is determined from representative TEM images of quenched samples. The microscopic stress, or micro-stress $\left(\sigma_{\mathrm{m}}\right)$, is calculated as $\sigma_{\mathrm{m}}=\varepsilon_{m} \times Y$, where $Y$ is the Young's modulus of $\mathrm{cBN}\left(909 \mathrm{GPa}^{53}\right)$. The differential micro-stress is defined as the difference between the micro-stresses derived from the detector elements at azimuth angles of $0^{\circ}$ and $90^{\circ}$, respectively. The macroscopic differential stress $(t)$, or macro-stress, defined as $t=\sigma_{1}-\sigma_{3}$ where $\sigma_{1}$ and $\sigma_{3}$ are axial and radial stress, respectively, differs from the differential micro-stress and is manifested by the variations in lattice strain detected by all detectors situated along azimuth angle $\psi$. Based on the results of lattice strain theory developed by Singh et $a .^{26,54,55}$, the macro-stress can be expressed as following equation:

$$
t(h k l)=6\langle Q(h k l)\rangle G(h k l)
$$

where $\langle Q(h k l)\rangle$ denotes the average $Q(h k l)$ value over all observed lattice reflections, and $G(h k l)$ is the aggregate shear modulus of polycrystalline sample for lattice plane $h k l$, which can be derived from single-crystal elastic constants for $\mathrm{cBN}$. Equation 4 can be further expressed as:

$$
d_{m}(h k l)=d_{p}(h k l)\left[1+\frac{t(h k l)}{6 G(h k l)}\left(1-3 \cos ^{2} \psi\right)\right]
$$


where $d_{m}(h k l)$ is the $d$-spacing of $(h k l)$ surveyed as a function of azimuth angel $\psi, d_{p}(h k l)$ is the $d$-spacing under hydrostatic pressure. By fitting the variation of $d_{m}(h k l)$ versus $\left(1-3 \cos ^{2} \psi\right)$ for $\mathrm{cBN}(111)$ peak (Fig. 1d-f) according to Eq. 5, the macro-stress can be determined from the slope ${ }^{56}$. All fittings to data collected at different pressure and temperature are presented in Fig. S2 (3.5 GPa/RT), Fig. S3 (3.5 $\left.\mathrm{GPa} / 1000{ }^{\circ} \mathrm{C}\right)$, and Fig. S4 $\left(4.0 \mathrm{GPa} / 1200^{\circ} \mathrm{C}\right)$. The resultant macro-stresses and differential micro-stresses at various conditions as a function of strain are shown in Fig. 2.

Stress-strain relations at RT As shown in Fig. 2a, the sample was already under negative macro-stress (i.e., $t<0 ; \sigma_{1}<\sigma_{3}$ ) at the onset of deformation at $3.5 \mathrm{GPa}$ and RT (3.5 GPa/RT). The macro-stress was generated due to non-cubic cavity in D-DIA during the cold compression to the target load with a nominal pressure of $3.5 \mathrm{GPa}$. The stress-strain curve exhibits a stepped variation at $\sim 3 \%$ strain. This is attributed to the brittle failure of larger grains in the sample, leading to grain-size reduction and subsequent cataclastic flow along grain boundaries, as evidenced by small nc-cBN grains of 2-17 nm forming serrated grain boundaries within large grains of 30-100 $\mathrm{nm}$ (Fig. S5a). Upon further deformation to an axial strain of $\sim 14 \%$, the macro-stress increased monotonically, reaching a total of $\sim 6.4 \mathrm{GPa}$ which is comparable to the stress accumulated in a micron-sized diamond sample deformed to nearly identical strain at similar conditions ${ }^{48}$, and no apparent yielding point was observed, demonstrating the superior strength of nano-grained cBN.

The micro-stresses derived from the peak-width analysis as a function of macro- 
strain are shown in Fig. $2 \mathrm{~b}$ for two azimuth angles, $0^{\circ}$ and $67.5^{\circ}$. It is evident that the micro-stresses at both angles experienced a step-variation, coincident with that of the macro-stress and in agreement with the notion that the brittle failures affect both external and local stresses. Similar to the macro-stress, the micro-stress at $0^{\circ}$ increased with increasing axial strain. In contrast, the micro-stress at $67.5^{\circ}$ remained more or less a constant, or even slightly down as the sample was deformed. This is due to the fact that, to hold the press load at a constant for the entirety of deformational process (i.e., for a constant pressure), the anvils at radial direction had to be slowly retracted (i.e., to reduce the radial stress) to accommodate the increase in axial stress.

The calculated differential micro-stresses are plotted in Fig. S6 for comparison with the macro-stresses in Fig. 2a. It is quite remarkable that the two stresses that are deduced from completely different methods agree well within experimental uncertainties, especially between $\sim 6-14 \%$ strain, i.e., after the brittle failure during the early stage of cold deformation. If the differential micro-stress is used as a proxy for the macro-stress $t$ in the sample, then according to Equation 5, the shear modulus of the sample can be extracted from the diffraction data recorded by the multi-element detector. Therefore, this practice could be used as a new method to constrain the elastic properties of crystalline solids at high pressures, greatly mitigating the lack of routine techniques to measure the shear modulus of solids.

Stress-strain relations at elevated temperatures For macro-stresses at high temperatures (Fig. 2a), the initial stage of stress-strain variation at both temperatures 
is similar to that of room-temperature in which the stress experienced a linear increase with increasing strain, i.e., as elastic deformation. However, the elastic region ended at much lower strain $\left(\sim 6 \%\right.$ at $1000^{\circ} \mathrm{C}$ and $\sim 4 \%$ at $1200{ }^{\circ} \mathrm{C}$; for comparison, $>14 \%$ at RT). Steady-state plastic deformation (i.e., relatively constant stress with increasing strain) was observed at both high temperatures. The higher the temperature, the lower the steady-state stress ( $\sim 5.8 \mathrm{GPa}$ for $1000{ }^{\circ} \mathrm{C}$ vs $\sim 3.8 \mathrm{GPa}$ for $\left.1200{ }^{\circ} \mathrm{C}\right)$. Fig. $2 \mathrm{c}$ and Fig. $2 \mathrm{~d}$ show the micro stress-strain relationships at $3.5 \mathrm{GPa} / 1000{ }^{\circ} \mathrm{C}$ and 4.0 $\mathrm{GPa} / 1200{ }^{\circ} \mathrm{C}$, respectively. It is evident that the micro stresses at elevated temperatures, unlike those at RT, rather quickly reached steady-state values, revealing the yielding and plastic deformation at grain contacts in the sample. For differential micro-stresses at both temperatures, similar to the observations at RT, the stress-strain relationship again largely agrees with those of macro-stress (see Figure S6). At each high temperature, the macro- and micro-stress deviated at the beginning of deformation. This is primarily due to the differences in stress relaxation between the sample and surrounding materials.

TEM characterization and microstructures analyses of the sample deformed at RT The structure and stability of the dislocations are of vital importance for understanding the extreme hardness and deformational properties of $\mathrm{cBN}$. To further elucidate the deformation mechanism of nc-cBN at atomic scale, we have carefully examined the recovered samples deformed at various experimental conditions using TEM. As shown in the bright-field TEM image of the sample deformed at RT in Fig. 3a, small nc particles (marked by red dashed arrows) distribute between much larger 
grains, a typical feature of cataclastic flows driven by GB sliding and rotation, indicating a dominant mechanism of brittle fracture and mechanic crushing for the deformation of nc-cBN at RT (see Fig. S5a). The fracture traces along (111) cleavages cutting through large grains are also manifested as nanocracks emitting from the surface of grains (Fig. 3b), as in bulk diamond ${ }^{48}$, which is an important source for nanoscale deformation and high-density dislocation. The recovered cBN sample has an average grain-size of $\sim 20 \mathrm{~nm}$. In contrast, the average grain size is $\sim 37$ $\mathrm{nm}$ for the starting materials (Figs. S7a). These measurements demonstrate a significant grain-size reduction during deformation and are consistent with the notion of fracturing and crushing induced by the grain rotation as the dominant deformation mechanism in nc-cBN at high pressure and RT.

The samples were further analyzed at atomic scale via high-resolution survey for dislocations, as shown in Fig. 3c. While only few edge dislocations existed in starting raw materials (see Figs. S7b and c), the wedge-like structures, amorphous regions and grains separated by high-angle grain boundaries (GBs) on the (111) plane were observed in the deformed sample. These features are surprisingly different from the twins and screw dislocations found in other diamond-structured materials deformed at similar conditions, such as diamond and silicon $(\mathrm{Si})^{48,57}$. The Debye rings in the inset of Fig. 3c can be attributed to nc-cBN; no other secondary phases were found. Shown in Fig. 3d is an inverse fast Fourier-filtered (IFFT) image depicting nc regions separated by abundant low-angle grain boundaries induced by applied stress - robust structures that could make $\mathrm{cBN}$ bulk stronger and harder. Figure $3 \mathrm{e}$ is an enlarged 
IFFT image of area in Fig. 3d, displaying two captive dislocations on (11̄1) and (111), both of which have extra half planes, meet to form the Lomer dislocations ${ }^{58-62}$. It is noted here that Lomer-Cottrell (L-C) locks are observed experimentally in superhard materials for the first time in the current study. The two white rectangular frames mark two L-C locks in Fig. 3d. We draw the Burgers circuits enclosing the core of the dislocations to confirm the Burgers vector of a $\frac{1}{2}(\overline{1} 10)$ full dislocation, which originates from the interaction of two non-dissociated $60^{\circ}$ full dislocations with Burgers vectors $\frac{1}{2}(\overline{1} 01)$ and $\frac{1}{2}(01 \overline{1})$, respectively. The dislocation reaction

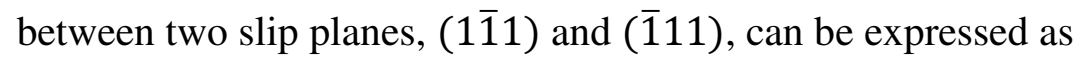

$$
\frac{1}{2}(\overline{1} 01)+\frac{1}{2}(01 \overline{1}) \rightarrow \frac{1}{2}(\overline{1} 10)
$$

Each of the two $60^{\circ}$ full dislocations can evolve into Shockley partial dislocations, one leading and one trailing. Subsequently, the stair-rod dislocations with a stable low energy configuration are formed by the reaction between the two leading Shockley partials, leading to sessile and immobile barriers against other dislocations in the slip plane ${ }^{59-62}$. With the increase of strain, low-angle dislocations continue to produce nano GBs and L-C locks, which in turn hinder the propagation of dislocations and slip of lattice planes and prevent the plastic deformation in $\mathrm{nc}-\mathrm{cBN}$ at strain up to at least $14 \%$. Hence, full dislocations dominate the elastic deformation activities of nc-cBN at 3.5 GPa and RT, which is in consistence with the results from dynamic simulation and indentation experiment of diamond ${ }^{41-44,61}$.

Figure $3 \mathrm{f}$ exhibits an enlarged wedge-like transition region surrounded by several grains shown in Fig. 3c. These features, produced by GB twisting induced by grain 
rotation, are in fact super-lattice-like structures with large spacing. The wedge-shaped lattice planes $\{111\}$ have rotated $\sim 20.7^{\circ}$ when compared to the original planes, suggesting that the super-lattice-like structure remains with Moiré patters. Based on the formula of Moiré patters fringe spacing:

$$
D=\frac{d}{2 \sin \frac{\theta}{2}}
$$

where $D$, equaling to $5.219 / 9=0.5799 \mathrm{~nm}$, is the Moiré fringe spacing, and $d$ represents the lattice spacing of the twisting crystal plane, $d=d_{111}=0.2088 \mathrm{~nm}$, the twisting angle $\theta$ (owing to the angular mismatch of lattice orientation) can be calculated to be $20.7^{\circ}$, which is a good agreement with the rotation angle of $20^{\circ}$ measured directly on the HRTEM image. It is thus concluded that, at $3.5 \mathrm{GPa}$ and RT, the deformation of $\mathrm{cBN}$ is dominated by brittle fracture and mechanic crushing during grain rotations mediated by full dislocations.

\section{TEM characterization and microstructures analyses at elevated temperature}

The microstructure of the recovered sample deformed at $3.5 \mathrm{GPa} / 1000{ }^{\circ} \mathrm{C}$ was analyzed to explore the dominant deformation mechanism at high temperature. Bright-field TEM images show fuzzy grain boundaries (see Fig. S5b). Majority of grains have diameters below $30 \mathrm{~nm}$, with an average value of $\sim 25 \mathrm{~nm}$, indicating that dynamic recrystallization induced by plastic deformation occurred at these conditions. Unlike deformation by brittle fracture and mechanic crushing at RT, plastic deformation mediated by GB twisting and grain rotation dominated the deformation behavior of nc-cBN, as shown in Fig. 4a, since high temperature can effectively relax the accumulated stress and reduce dislocation pileup. Super-lattice-like patterns 
(marked with red dashed circles) are the primary features of GB twisting and grain rotation close to grain boundaries (also see Fig. S8). It is worth noting that for the first time plastic deformation mediated by grain rotations near GBs is observed experimentally in superhard nc-cBN. The wide distribution of twisting angle $\theta\left(10^{\circ}-\right.$ $34^{\circ}$ ) among different Moiré patterns indicates nc-cBN grains have varying degrees of rotation depending on the local shear stress. The Debye rings in the inset of Fig. 4a can be largely attributed to nc-cBN, but with an extra ring having a lattice $d$-spacing of $\sim 0.358 \mathrm{~nm}$, which is larger than the interplanar spacing $\sim 0.345 \mathrm{~nm}$ of (002) of hBN. XRD patterns (Fig. S1) however suggest that no other secondary phases were present, indicating that this extra ring is related to super-lattice-like Moiré fringes. Through careful examination of the trailing region of rotated grains near GB, highdensity dislocations were found, as shown in Fig. $4 \mathrm{~b}$. Burgers circuits are drawn to show it is a $60^{\circ}$ full dislocation because only one set of (111) planes possess an extra half plane, which is different from the combined full dislocation with a Burgers vector along $\frac{1}{2}(\overline{1} 10)$ at RT since high temperature breaks the L-C locks (Fig. 4c). These $60^{\circ}$ full dislocations as $\frac{1}{2}(\overline{1} 01)$ on $(1 \overline{1} 1)$ plane can be dissociated into two $90^{\circ}$ and $30^{\circ}$ Shockley partial dislocations: a leading and trailing dislocation as follows:

$$
\frac{1}{2}(\overline{1} 01) \rightarrow \frac{1}{6}(\overline{1} 12)+\frac{1}{6}(\overline{2} 11)
$$

Thus, the plastic deformation of nc-cBN was mainly mediated by $90^{\circ}$ and $30^{\circ}$ Shockley partial dislocations near twisting GBs. Twin dislocations and other mixed dislocation interactions should also be responsible for plastic deformation of nc-cBN, as shown in Fig. 4d. The formation of twins is closely related to the stacking faults. 
Obviously, $\{111\}$ lattice planes can provide a mirror symmetry of the directional relationship in each side of stacking faults. High shear stress is likely the reason for the formation of deformation twins. Examination of Fig. 4e reveals Burgers circuit encloses a $90^{\circ}$ Shockley partial dislocation with a Burgers vector along $\frac{1}{6}(11 \overline{2})$ near the stacking faults. These dislocations produced by grain rotation and stacking faults are intermediaries supported by (111) planes and thus a source of plastic deformation.

Bright-field TEM image of the sample deformed at $4.0 \mathrm{GPa}$ and $1200{ }^{\circ} \mathrm{C}$ shows clear and smooth grain boundaries as a result of dynamic recrystallization during plastic flow (Fig. 5a, also see Fig. S5c). Majority of grains have a diameter below 30 $\mathrm{nm}$, with an average value of $\sim 27 \mathrm{~nm}$. Similar to $\mathrm{nc}-\mathrm{cBN}$ deformed at 3.5 $\mathrm{GPa} / 1000{ }^{\circ} \mathrm{C}$, the deformation mechanism at $1200{ }^{\circ} \mathrm{C}$ is dominated by plastic flow/creep which was however mediated by phase transitions (Fig. 5b-f). The traces of GB twisting were observed on planes with a $d$-spacing of $0.338 \mathrm{~nm}$ (Fig. 5b), suggesting the presence of $\mathrm{hBN}$. As no hBN or wurtzite $\mathrm{BN}(\mathrm{wBN})$ impurities were detected by XRD in the starting materials (Fig. S9), it is inferred that cBN was directly converted to hBN due to shear-induced phase transition. Likewise, superlattice-like structures evolved from the original GB twisting have also been observed in SAED (inset, Fig. 5a) and FFT (Fig. 5e). Figure 5f and its FFT inset show some superlattices with $d$-spacing of $0.2189 \mathrm{~nm}$ which may belong to the (100) lattice plane of $\mathrm{wBN}$. Based on the pressure-shear experiments in the rotational diamond anvil cell $(\mathrm{RDAC})^{45}$, plastic shear can induce phase transition under an applied pressure 3-20 times lower than the equilibrium phase-transition pressure ${ }^{45,46}$. Low-strength hBN 
and $\mathrm{wBN}$ as precipitated phases close to the grain boundaries help relax the accumulated stress. Generally, pressure-induced phase transitions originate from existing defects, whereas strain-induced phase transitions take place at new defects formed during deformation. Shear-dominated stress tensor may result in new phase transformation paths to relax concentrated stress ${ }^{45,63}$. Hence, understanding the deformation process and evolution of dislocations is conducive to designing nc superhard materials with superior mechanical and physical properties.

\section{Conclusion}

In summary, the inherent strain-stress relations and evolution of the deformation mechanisms of nc-cBN have been systematically investigated using a D-DIA largevolume apparatus, in situ XRD measurements at high pressure and temperature, and HRTEM. We found the differential micro-stresses derived from peak-broadening analysis can potentially be used as proxies for the macro-stresses defined by lattice strains. The microstructures analyses suggest the room-temperature deformation of $\mathrm{nc}-\mathrm{cBN}$ is dominated by brittle fracture and mechanic crushing induced by grain rotation which is mediated by combined full dislocations. The formation of L-C locks has been observed experimentally for the first time in the deformed superhard nc$\mathrm{cBN}$. At $1000{ }^{\circ} \mathrm{C}$, plasticity of nc-cBN is dominated by grain rotation and twinning mediated by Shockley partial dislocations. Shear-dominated stress tensor at grain boundaries leads to new phase-transformation paths of $\mathrm{cBN}$ to $\mathrm{hBN}$ to mitigate the concentrated stress at $1200^{\circ} \mathrm{C}$. These observations shed light on the critical roles of dislocation in elasticity, plasticity and creep of nc superhard materials. 


\section{Methods}

In situ high-pressure deformation experiments and HRTEM characterizations. High-pressure and high-temperature deformation experiments in conjunction with in situ energy-dispersive x-ray diffraction (XRD) were conducted at 6-BMB beamline station, Advanced Photon Source (APS), Argonne National Laboratory (ANL) on nc-cBN with an average size of $40 \mathrm{~nm}$ packed in an hBN capsule about $6.2 \mathrm{~mm}$ long and $1.2 \mathrm{~mm}$ in diameter using a D-DIA apparatus and a $100 \times 200 \mu \mathrm{m}$-ray beam $^{47}$. One sintered polycrystalline diamond (PCD) anvil was used for the multi-element detector to receive the diffracted $\mathrm{x}$-rays ${ }^{48,52}$. Energy-dispersive XRD data were collected with a counting-time of $\sim 10$ minutes using a ten-element Ge solid-state detector (SSD) on which each element is located at a fixed azimuth angle $\psi\left(22.5^{\circ}\right.$ apart from $0^{\circ}-180^{\circ}$, plus one at $\left.270^{\circ}\right)$. To eliminate diffraction signals not from the sample, a conical-slit system located between the sample and the detectors was employed to collimate and constrain the $2 \theta$ angle of the diffracted x-rays. Radiographic images were taken, with data collection toggling continuously between diffraction and imaging modes. An amorphous boronepoxy cube was used as pressure medium for deformation experiments. Dense $\mathrm{Al}_{2} \mathrm{O}_{3}$ pistons were placed at both ends of specimens, which were pre-compressed into pellets and packed into a hexagonal boron nitride $(\mathrm{hBN})$ capsule; three $25-\mu \mathrm{m}$-thick tantalum disks were placed at the piston-specimen and specimen-specimen contacts as strain markers. The nc-cBN sample was first compressed to desired pressure at RT and then annealed at high temperature $\left(\sim 1000^{\circ} \mathrm{C}\right)$ for 1 hour using enclosed resistive graphite heater. As the detector elements at the azimuth angle of $90^{\circ}$ and $270^{\circ}$ (radial direction) weren't available during all experiments, signals recorded by the element situated at $67.5^{\circ}$ are taken as the proxies for those from the radial direction in this study. Furthermore, because diffraction peaks (200), (220) and (311) were either very weak or even absent during high-pressure deformational 
experiments, the stress analysis in this study is based on the diffraction peak (111) only. The recovered samples were processed by focused ion beam (FIB) technique, and the chemical composition and microstructures have been analyzed using high-resolution transmission electron microscopy (HRTEM) at the Center for Functional Nanomaterials, Brookhaven National Laboratory. 


\section{REFERENCES}

(1) Tian, Y. J. et al. Ultrahard nanotwinned cubic boron nitride. Nature 493, 385$388(2013)$.

(2) Solozhenko, V. L., Kurakevych, O. O. \& Le Godec, Y. Creation of nanostuctures by extreme conditions: high-pressure synthesis of ultrahard nanocrystalline cubic boron nitride. Adv. Mater. 24, 1540-1544 (2012)

(3) Dubrovinskaia, N. et al. Superhard nanocomposite of dense polymorphs of boron nitride: noncarbon material has reached diamond hardness. Appl. Phys. Lett. 90, 101912 (2007).

(4) Sumiya, H., Uesaka, S. \& Satoh, S. Mechanical properties of high purity polycrystalline cBN synthesized by direct conversion sintering method. $J$. Mater. Sci. 35, 1181-1186 (2000).

(5) Liu, G. et al. Submicron cubic boron nitride as hard as diamond. Appl. Phys. Lett.106, 121901 (2015).

(6) Mirkarimi P. B., McCarty K. F., Medlin D. L., Review of advances in cubic boron nitride film synthesis. Mater. Sci. Eng. R Rep. 21, 47-100 (1997).

(7) Solozhenko, V. L., Andrault, D., Fiquet, G., Mezouar, M., \& Rubie, D. C. Synthesis of superhard cubic $\mathrm{BC}_{2} \mathrm{~N}$. Appl. Phys. Lett. 78, 1385-1387 (2001).

(8) Wang, P. et al. Diamond-c BN alloy: A universal cutting material. Appl. Phys. Lett. 107, 101901 (2015).

(9) Wentorf, R. H. Jr., Cubic form of boron nitride. J. Chem. Phys. 26, 956 (1957).

(10) Solozhenko V. L. and Turkevich, V. Z. Thermoanalytical study of the 
polymorphic transformation of cubic into graphite-like boron nitride. $J$. Therm. Anal. 38, 1181 (1992).

(11) Wuchina, E., Opila, E., Opeka, M., Fahrenholtz, W., \& Talmy, I. UHTCs: ultra-high temperature ceramic materials for extreme environment applications. Electrochem. Soc. Interface. 16, 30 (2007).

(12) Solozhenko, V. L., Turkevich, V. Z., \& Holzapfel, W. B. Refined phase diagram of boron nitride. J. Phys. Chem. B 103, 2903-2905(1999).

(13) Shipilo, V. B., Leushkina, G. V., Makovetskaya, L. A., \& Popel'nyuk, G. P. Electrical and thermal conductivity of beta-BN. Inorg. Mater. 22, 361-365 (1986).

(14) Yates, B., Overy, M. J., \& Pirgon, O. The anisotropic thermal expansion of boron nitride: I. experimental results and their analysis. Philos. Mag. 32, 847857 (1975).

(15) Wang, Z. G., Wong, Y. S., and Rahman M., High-speed milling of titanium alloys using binderless CBN tools. Int. J. Mach. Tool Manufact. 45, 105-114 (2005).

(16) Roundy, D. \& Cohen, M. L. Ideal strength of diamond, Si, and Ge. Phys. Rev. B 64, 212103 (2001).

(17) Luo, X., Liu, Z., Xu, B., Yu, D., Tian, Y., Wang, H. \& He, J. Compressive Strength of Diamond from First-Principles Calculation. J. Phys. Chem. C 114, 17851-17853 (2010).

(18) Telling, R. H., Pickard, C. J., Payne, M. C. \& Field, J. E. Theoretical Strength 
and Cleavage of Diamond. Phys. Rev. Lett. 84, 5160-5163 (2000).

(19) Casanova, C. A. M., Balzaretti, N. M., Voronin, G., \& Da Jornada, J. A. H. Experimental study of plastic deformation during sintering of cubic boron nitride compacts. Diam. Relat. Mater. 8, 1451-1454 (1999).

(20) Evens, T., Wild, R. K. \& Thomson, J. J. Plastic Deformation of Diamond at Temperatures below $1800{ }^{\circ}$ C. Philos. Mag. 13, 209-210 (1965).

(21) Field, J. E. \& Pickles, C. S. J. Strength, fracture and friction properties of diamond. Diam. Relat. Mater. 5, 625-634 (1996).

(22) Mao, H. K. \& Hemley, R. J. Optical transitions in diamond at ultrahigh pressures. Nature 351, 721-724 (1991).

(23) Akahama, Y. \& Kawamura, H. Raman study on the stress state of [111] diamond anvils at multimegabar pressure. J. Appl. Phys. 98, 083523-083526 (2005).

(24) Hemley, R. J., Mao, H. K., Shen, G., Badro, J., Gillet, P., Hanfland, M. \& Hausermann, D. X-ray Imaging of Stress and Strain of Diamond, Iron, and Tungsten at Megabar Pressures. Science 276, 1242-1245 (1997).

(25) Eremets, M. I., Trojan, I. A., Gwaze, P., Huth, J., Boehler, R. \& Blank, V. D. The strength of diamond. Appl. Phys. Lett. 87, 141902 (2005).

(26) He, D. W. Shieh, S. R. and Duffy, T. S. Strength and equation of state of boron suboxide from radial $\mathrm{x}$-ray diffraction in a diamond cell under nonhydrostatic compression. Phys. Rev. B 70, 184121 (2004).

(27) Zhao, Y. \& Zhang, J. Microstrain and grain-size analysis from diffraction peak 
width and graphical derivation of high-pressure thermomechanics. J. Appl. Crystallog. 41, 1095-1108 (2008).

(28) Zhang, J. Zhao, Y. \& Palosz, B. Comparative studies of compressibility between nanocrystalline and bulk nickel. Appl. Phys. Lett. 90, 043112 (2007).

(29) Wang, Y. Zhang, J. \& Zhao, Y. Strength weakening by nanocrystals in ceramic materials. Nano Lett. 7, 3196-3199 (2007).

(30) Weidner, D. J. Wang, Y. \& Vaughan, M. T. Strength of diamond. Science, 266, 419-422 (1994).

(31) Meyers M. A., Mishra A., Benson D. J., Mechanical properties of nanocrystalline materials, Prog. Mater. Sci. 51, 427-556 (2006).

(32) Poirier J. P. Creep of crystals: high-temperature deformation processes in metals, ceramics and minerals. Cambridge University Press, 1985.

(33) Zhu, Y. T. Liao, X. Z. Wu, X. L. Deformation twinning in nanocrystalline materials. Prog. Mater. Sci. 57, 1-62 (2012).

(34) Hu, J. Shi, Y. N. Sauvage, X. Sha, G. Lu. K. Grain boundary stability governs hardening and softening in extremely fine nanograined metals. Science $\mathbf{3 3 5}$. 1292-1296 (2017).

(35) Cheng, Z. Zhou, H. F. Lu, Q. H. Gao, H. J. Lu, L. Extra strengthening and work hardening in gradient nanotwinned metals. Science 362. 6414 (2018).

(36) Chen, M. W. et al. Deformation Twinning in Nanocrystalline Aluminum. Science 300, 1275-1277 (2003).

(37) Lehtinen, O. Kurasch, S. Krasheninnikov, A. V. \& Kaiser, U. Atomic scale 
study of the life cycle of a dislocation in graphene from birth to annihilation. Nat. Commun. 4, 2098 (2013).

(38) Huang, Q. et al. Nanotwinned diamond with unprecedented hardness and stability. Nature 510, 250 (2014).

(39) Wang, L. et al. Grain rotation mediated by grain boundary dislocations in nanocrystalline platinum. Nat. Commun. 5, 4402 (2014).

(40) Hovsepian, P. E., Lewis, D. B., \& Münz, W. D. Recent progress in large scale manufacturing of multilayer/superlattice hard coatings. Surf. Coat. Technol. 133, 166-175 (2000).

(41) Alexander, H. \& Haasen, P. Dislocations and plastic flow in the diamond structure. Solid State Phys. 22, 27-158 (1969).

(42) Xiao, J. W. et al. Dislocation behaviors in nanotwinned diamond. Sci. Adv. 4, 8195 (2018).

(43) Blase, X. Lin, K. Canning, A. Louie, S. G. \& Chrzan, D. C. Structure and energy of the $90^{\circ}$ partial dislocation in diamond: A combined $A b$ initio and elasticity theory analysis. Phys. Rev. Lett. 84, 5780 (2000).

(44) Read, W. T. \& Shockley, W. Dislocation models of crystal grain boundaries. Phys. Rev. 78, 275 (1950).

(45) Levitas, V. I. \& Javanbakht, M. Phase transformations in nanograin materials under high pressure and plastic shear: nanoscale mechanisms. Nanoscale 6, $162-166$ (2014).

(46) Ji, C. et al. Shear-induced phase transition of nanocrystalline hexagonal boron 
nitride to wurtzitic structure at RT and lower pressure. Proc. Natl. Acad. Sci. USA. 109, 19108-19112 (2012).

(47) Wang, Y., Durham, W. B., Getting, I. C., \& Weidner, D. J. The deformationDIA: A new apparatus for high temperature triaxial deformation to pressures up to 15 GPa. Rev. Sci. Instrum. 74, 3002-3011 (2003).

(48) Yu, X. et al. Constitutive law and flow mechanism in diamond deformation. Sci. Rep. 2, 876(2012).

(49) Wang, P. et al., Vanadium Diboride $\left(\mathrm{VB}_{2}\right)$ Synthesized at High Pressure: Elastic, Mechanical, Electronic, and Magnetic Properties and Thermal Stability. Inorg. Chem. 57, 1096-1105 (2018).

(50) Raterron, P. Merkel, S. \& Holyoke III, C. W. Axial temperature gradient and stress measurements in the deformation-DIA cell using alumina pistons. Rev. Sci. Instrum. 84, 043906 (2013).

(51) Raterron, P. Chen, J. Li, L. Weidner, D. \& Cordier, P. Pressure-induced slipsystem transition in forsterite: Single-crystal rheological properties at mantle pressure and temperature. Am. Mineral. 92, 1436-1445 (2007).

(52) Li, L. et al. Deformation of olivine at mantle pressure using the D-DIA. Eur. J. Mineral. 18, 7-19 (2006).

(53) Grimsditch, M. Zouboulis, E. S. \& Polian, A. Elastic constants of boron nitride. J. Appl. Phys. 76, 832-834 (1994).

(54) Singh, A. K. The lattice strains in a specimen (cubic system) compressed nonhydrostatically in an opposed anvil device. J. Appl. Phys. 73, 4278-4286 
(1993).

(55) Singh, A. K. Balasingh, C. Mao, H. K. Hemley, R. J. \& Shu, J. Analysis of lattice strains measured under nonhydrostatic pressure. J. Appl. Phys. 83, 7567-7575 (1998).

(56) Hutchinson, W. B. \& Barnett, M. R. Effective values of critical resolved shear stress for slip in polycrystalline magnesium and other hcp metals. Scr. Mater. 63, 737-740 (2010).

(57) Föll, H. \& Ast, D. TEM observations on grain boundaries in sintered silicon. Philos. Mag. A 40, 589-610 (1979).

(58) Bourret, A. Desseaux, J. \& Renault, A. Core structure of the Lomer dislocation in germanium and silicon. Philos. Mag. A 45, 1 (1982).

(59) Wang, L. Han, X. Liu, P. Yue, Y. Zhang, Z. and Ma, E. In Situ Observation of Dislocation Behavior in Nanometer Grains. Phys. Rev. Lett. 105, 135501 (2010).

(60) Bulatov, V. Abraham, F. F., Kubin, L., Devincre, B., \& Yip, S. Connecting atomistic and mesoscale simulations of crystal plasticity. Nature (London) 391, 669 (1998).

(61) Blumenau, A. T. Heggie, M. I. Fall, C. J. Jones, R. \& Frauenheim, T. Dislocations in diamond: Core structures and energies. Phys. Rev. B 65, 205205 (2002).

(62) Wu, X. L. Zhu, Y. T., Wei, Y. G., \& Wei, Q. Strong strain hardening in nanocrystalline nickel. Phys. Rev. Lett. 103, 205504 (2009). 
(63) Yue, B. et al. Deformation behavior across the zircon-scheelite phase transition. Phys. Rev. Lett. 117, 135701 (2016). 


\section{Acknowledgements}

This work is supported by the National Natural Science Foundation of China (Grant No. 52002166), the Shenzhen Engineering Research Center for Frontier Materials Synthesis at High Pressures, the Shenzhen Peacock Plan (No.

KQTD2016053019134356), the Guangdong Innovative \& Entrepreneurial Research Team Program (No. 2016ZT06C279), and the Shenzhen Novel Nano-Material Sciences and the Research Platform for Crystal Growth \& Thin-Film Preparation at SUSTech. The deformation experiments were performed at 6-BM-B (Sector 6), Advanced Photon Source (APS), Argonne National Laboratory, which is supported by the National Science Foundation-Earth Sciences (EAR1128799) and Department of Energy-GeoSciences (DEFG02-94ER14466). The Advanced Photon Source is a U.S. Department of Energy (DOE) Office of Science User Facility operated for the DOE Office of Science by Argonne National Laboratory under Contract No. DE-AC0206CH11357. K.K. acknowledges support to the Center for Functional Nanomaterials, which is a U.S. DOE Office of Science Facility, at Brookhaven National Laboratory under Contract No. DE-SC0012704.

\section{Author contributions}

P.W., L.P.W., and Y.S.Z. designed the project. P.W, H.Y.C. and L.P.W. performed the synchrotron x-ray diffraction experiments. K.K. conducted FIB and HRTEM experiments on recovered cBN samples. P.W. analyzed the data and wrote the draft. L.P.W. and H.Y.C. edited the manuscript. All authors discussed the results and provided inputs for data interpretation. 


\section{Supplementary Information}

Detailed sample characterizations (including XRD patterns of nc-cBN along the entire compression, annealing, and deformation path, the $d$-spacing of the (111) cBN peak fitted against azimuth angles at various strains, confining pressure and temperature, TEM, XRD pattern of deformed samples and raw material, and the calculated differential micro-stresses of nc-cBN sample deformed at $3.5 \mathrm{GPa} / \mathrm{rt}, 3.5$ $\mathrm{GPa} / 1000{ }^{\circ} \mathrm{C}$ and $4.0 \mathrm{GPa} / 1200{ }^{\circ} \mathrm{C}$ are plotted for comparison with the macrostresses).

\section{Additional information}

Supplementary Information accompanies this paper at http://www.nature.com/naturecommunications

Competing financial interests: The authors declare no competing financial interests. 


\section{Figure captions}

Figure 1. (Color online) Schematic diagrams of in situ high-pressure D-DIA experimental set-up (a) and cell assembly (b); evolution of the diffraction peak (111) of nc-cBN collected at azimuth angle of $180^{\circ}$ (c); and selected plots of lattice spacing vs azimuth angle for varying stages of deformation and determination of differential stresses (d-f).

Figure 2. (Color online) Macro differential stress and micro-stress of nc-cBN as functions of total axial strain at different pressure and temperature conditions. (a) Representative stress-strain curves of nc-cBN deformed at $3.5 \mathrm{GPa} / \mathrm{RT}$ (green diamond), $3.5 \mathrm{GPa} / 1000{ }^{\circ} \mathrm{C}$ (hollow red hexagon) and $4.0 \mathrm{GPa} / 1200{ }^{\circ} \mathrm{C}$ (blue sphere); micro-stresses determined from $0^{\circ}$ and $67.5^{\circ}$ azimuth angles for samples deformed at 3.5 GPa/RT (b), $3.5 \mathrm{GPa} / 1000{ }^{\circ} \mathrm{C}(\mathrm{c})$ and $4.0 \mathrm{GPa} / 1200{ }^{\circ} \mathrm{C}(\mathrm{d})$.

Figure 3. (Color online) TEM observations of nc-cBN deformed at $3.5 \mathrm{GPa}$ and RT. (a) Bright-field TEM image; red dashed arrows pointing to the crushed particles between larger grains. (b) Bright-field TEM image; green dashed arrows pointing to the propagating nanocracks. (c) HRTEM micrograph displaying various features in the deformed sample; see text for details. (d) IFFT image of low-angle dislocations on (111) lattice plane. White rectangular frames mark two Lomer-Cottrell (L-C) locks. (e) Enlarged image of the white rectangular frame in (d) showing the two extra half

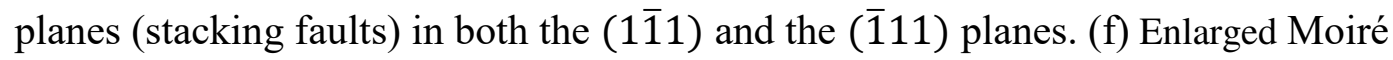
fringes marked in (c). The twisted grain boundaries are due to grain rotation. (g) Schematic drawing of twisted GB along (110) axis in (f). 
Figure 4. (Color online) HRTEM observations of dislocation movements in cBN sample deformed at $3.5 \mathrm{GPa}$ and $1000^{\circ} \mathrm{C}$. (a) The red circles and rectangular fields (Moiré pattern) showing active grain rotation near high-angle grain contacts. Twin boundary (stacking faults) along $\langle 111\rangle$ direction marked with white lines. The inset is a SAED of nc-cBN with a superlattice diffraction ring (100). (b) Representative Moiré pattern IFFT image of the area " $\mathrm{A}$ " in (a), indicating grain-rotation-induced stacking faults. (c) Enlarged Moiré image in (b). Burgers circuit enclosing a Shockley partial on $60^{\circ}$ full dislocation. Edge dislocations located at Moiré fringes are marked with "T". (d) Representative IFFT image of twinning for the area " $H$ " in (a), also showing grain-rotation-induced stacking faults. (e) Enlarged IFFT image of twinning structures of the area " $\mathrm{H}$ " in (a); Burgers circuit enclosing two Shockley partials.

Figure 5. (Color online) HRTEM images of dislocation movements in cBN sample deformed at $4.0 \mathrm{GPa}$ and $1200^{\circ} \mathrm{C}$. (a) Bright-field TEM image showing grain boundaries due to dynamic recrystallization of crushed grains under plastic flow. The inset is a SAED with an extra Debye ring of $0.338 \mathrm{~nm}$ in radius, indicating stress induced phase transition of $\mathrm{cBN}$ to $\mathrm{hBN}$. (b) The red dashed circles represent active grain-rotation-induced dislocations near serrated grain boundaries. (c) Characteristic Moiré pattern IFFT image of the area enlarged from (b) on hBN. (d) Characteristic Moiré pattern IFFT image on cBN. (e) FFT taken from (c), which shows the superlattice plane (001) with a $d$-spacing of $0.3445 \mathrm{~nm}$ in hBN. (f) Typical superlattice structure on the sample. The rhombus represents the smallest repeating 
unit of the superstructure. Inset is the FFT taken from superlattice structure showing various superlattice planes. 
(a)
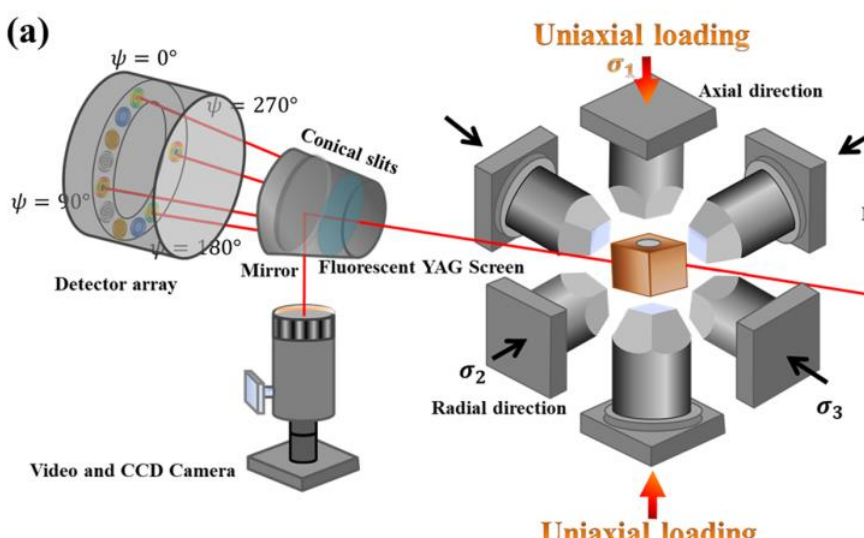

Motor-driven incident slits
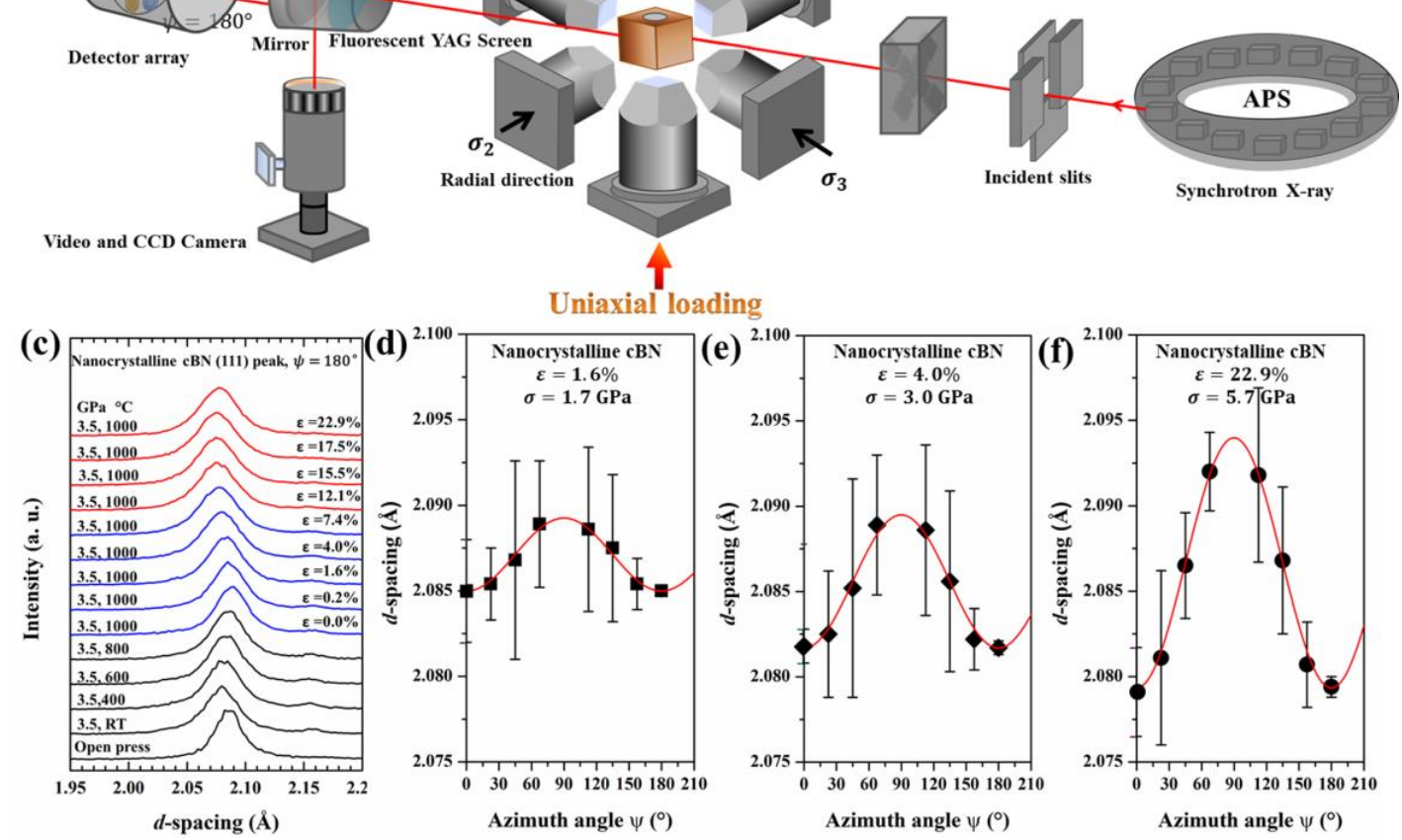

\section{Figure 1}



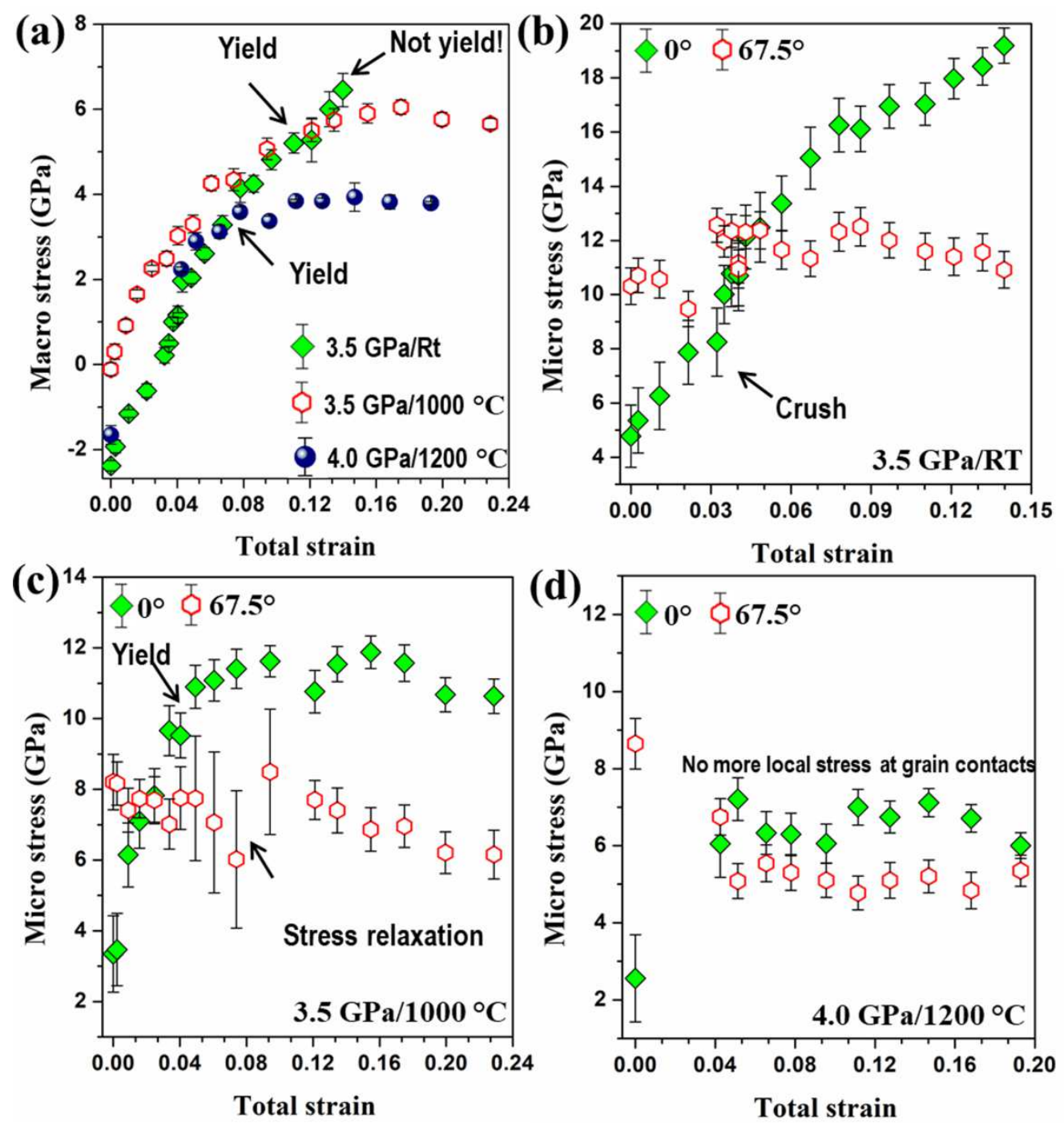

Figure 2 


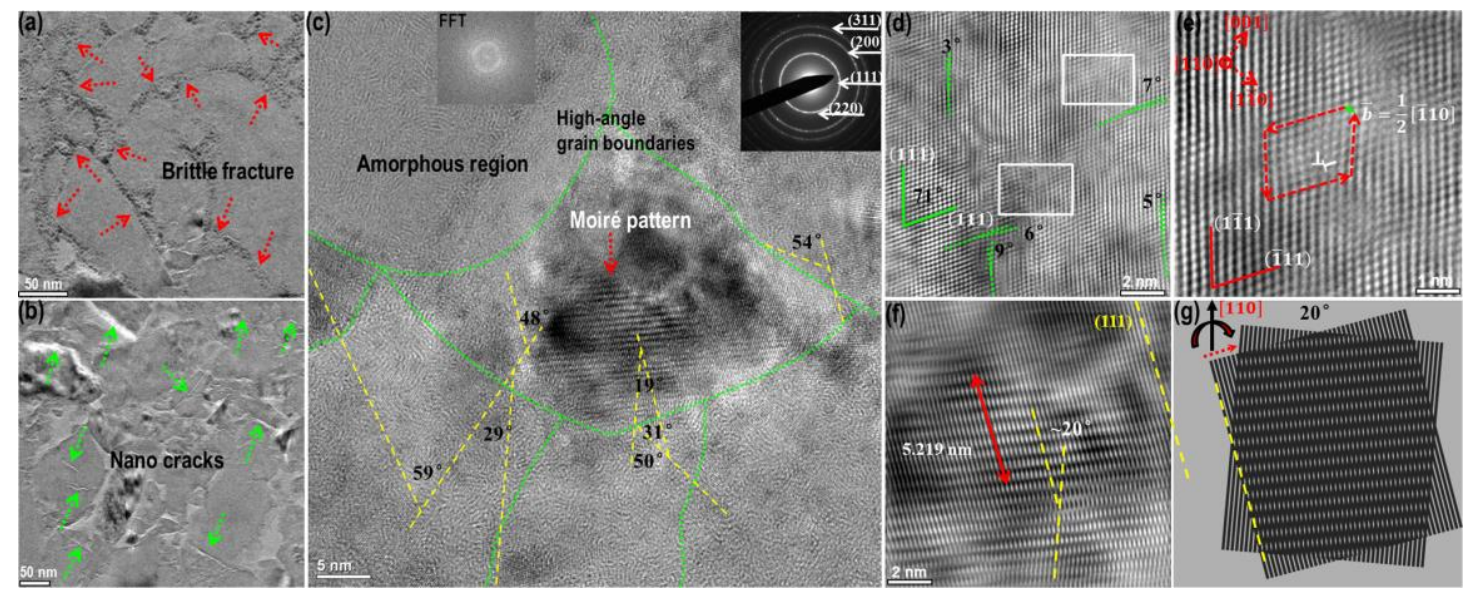

Figure 3 


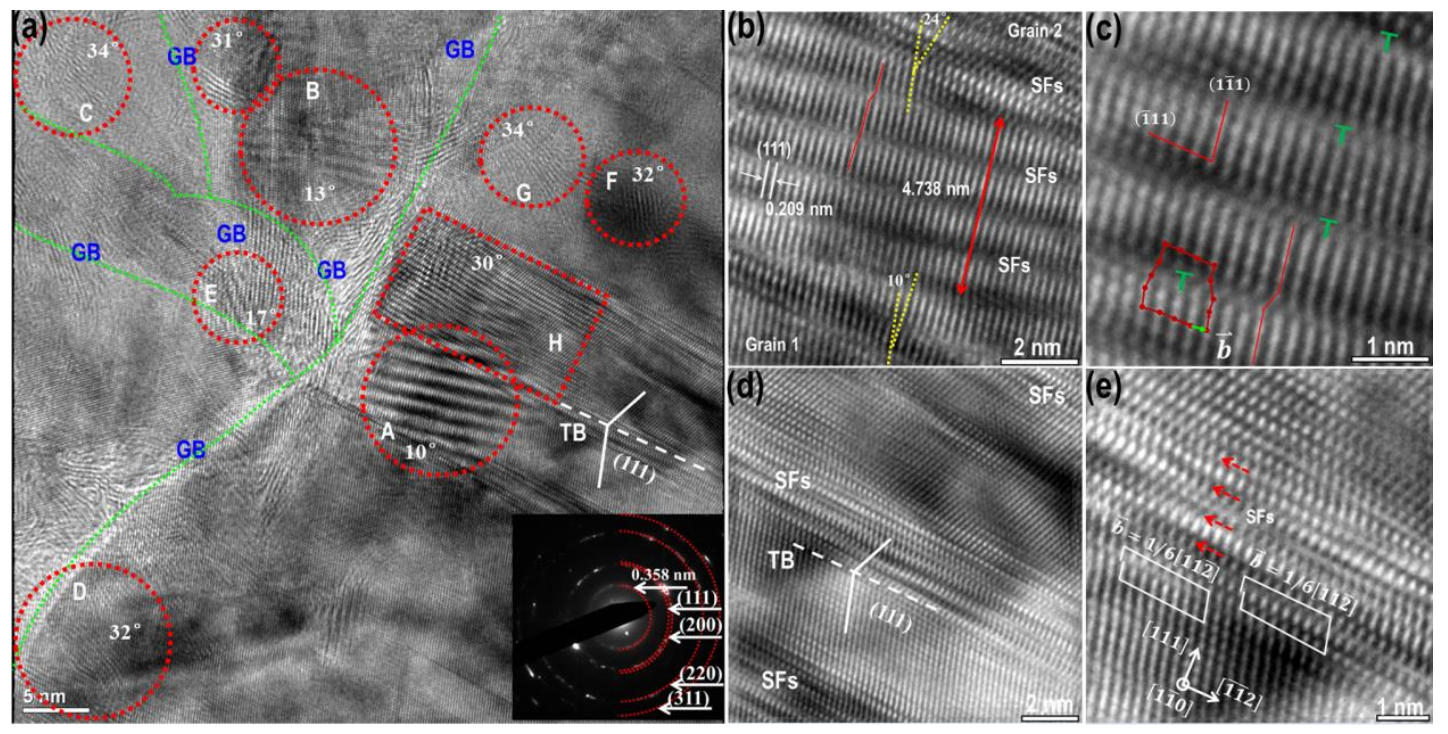

Figure 4 


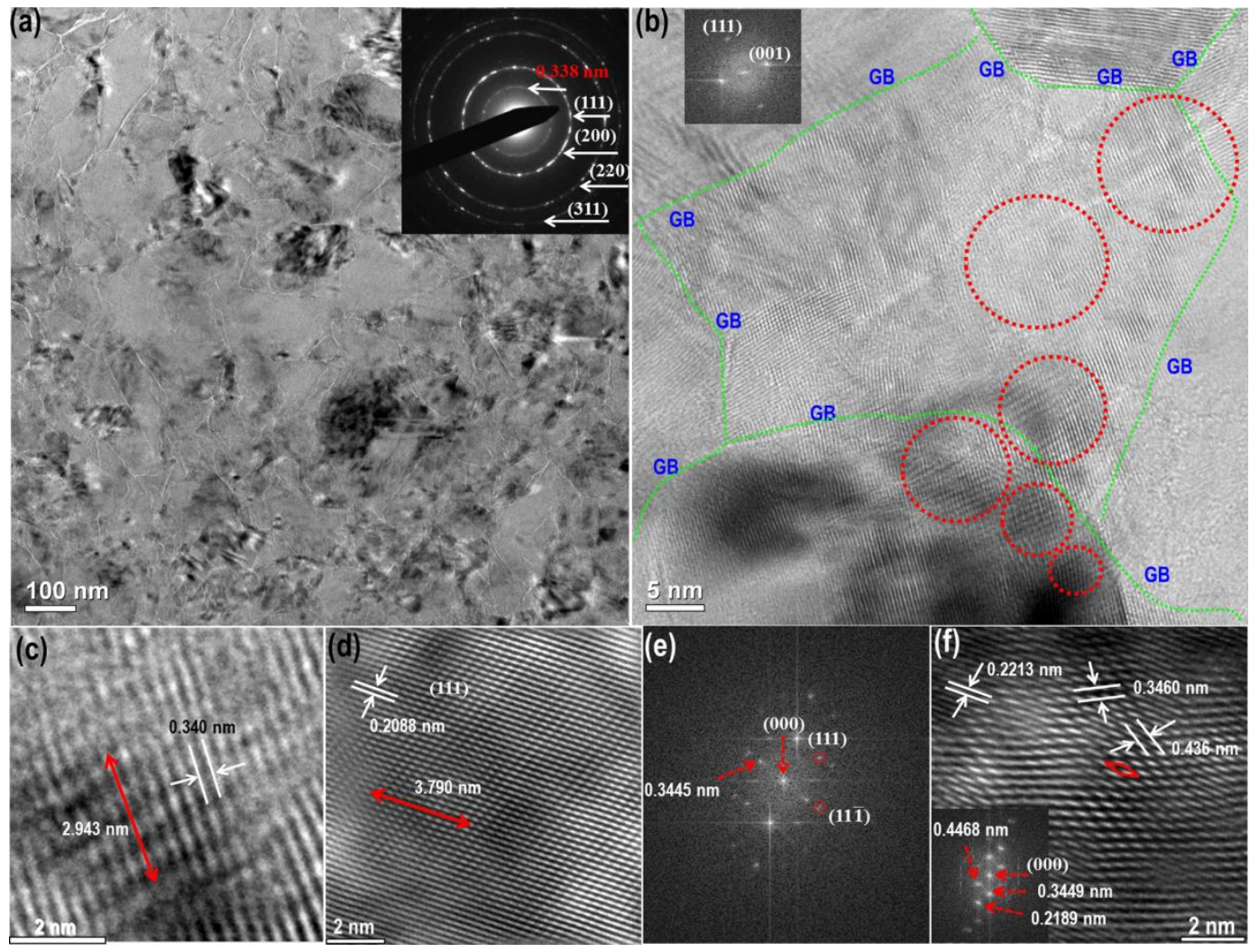

Figure 5 
(a)

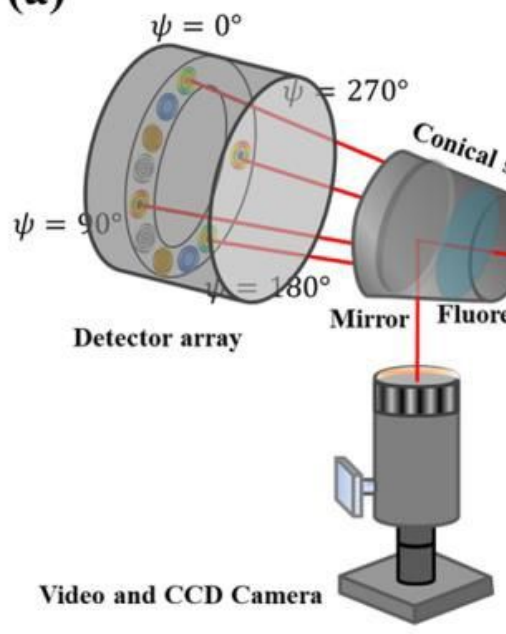

Uniaxial loading

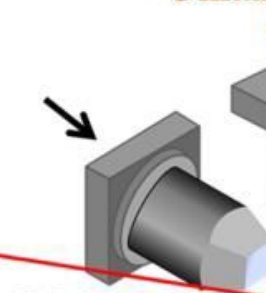

$\sigma_{1}$

(b)

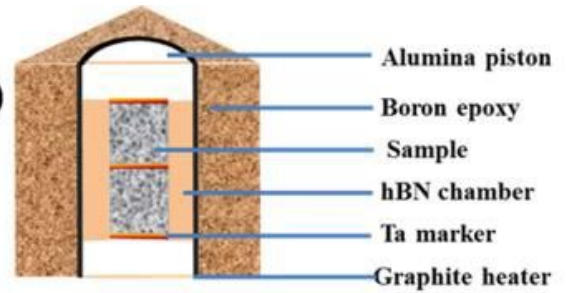

Motor-driven incident slits

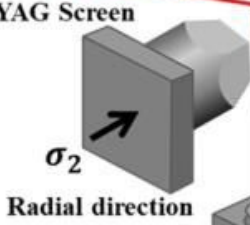

Axial direction

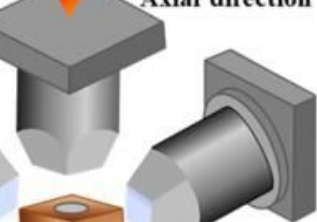

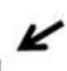

Graphite heater

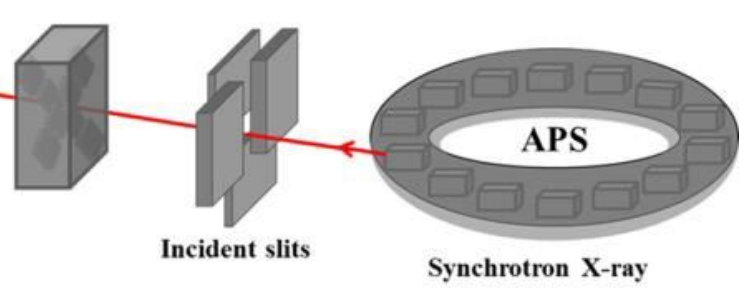

Radial direction

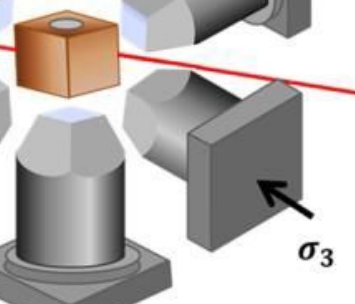

Uniaxial loading
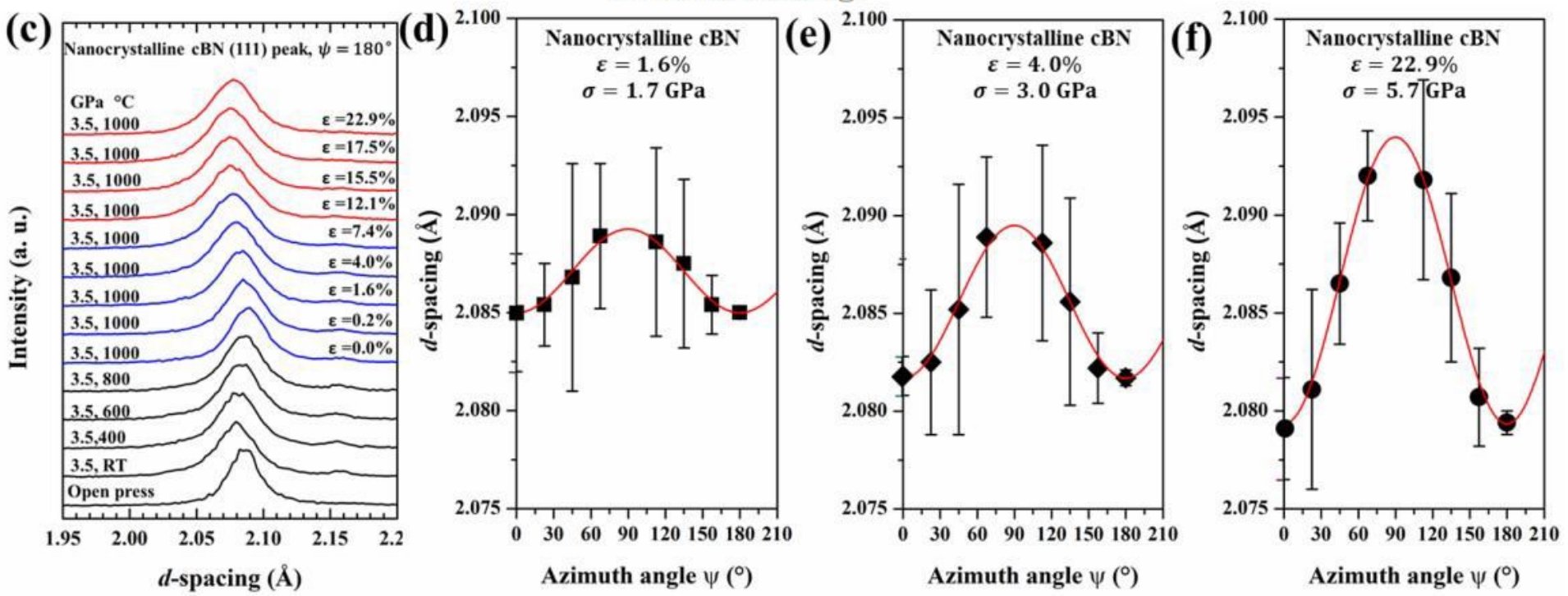

Figure 1

(Color online) Schematic diagrams of in situ high pressure D DIA experimental set up (a) and cell assembly (b); evolution of the diffraction peak (111) of nc cBN collected at azimuth angle of $180 \mathrm{o}$ (c); and selected plots of lattice spacing vs azimuth angle for varying stages of deformation and determination of differential stresses ( $d f$ ). 

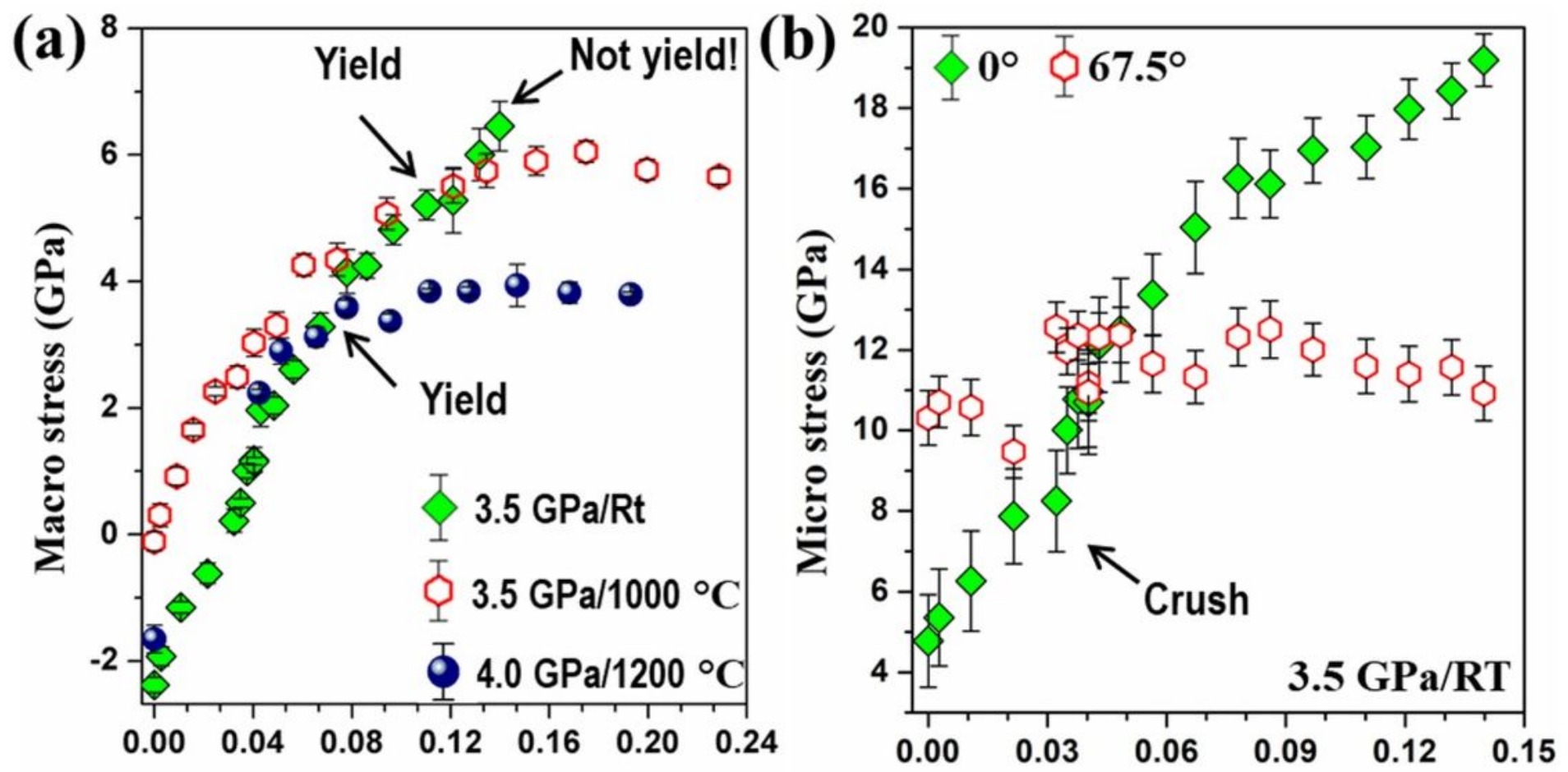

Total strain
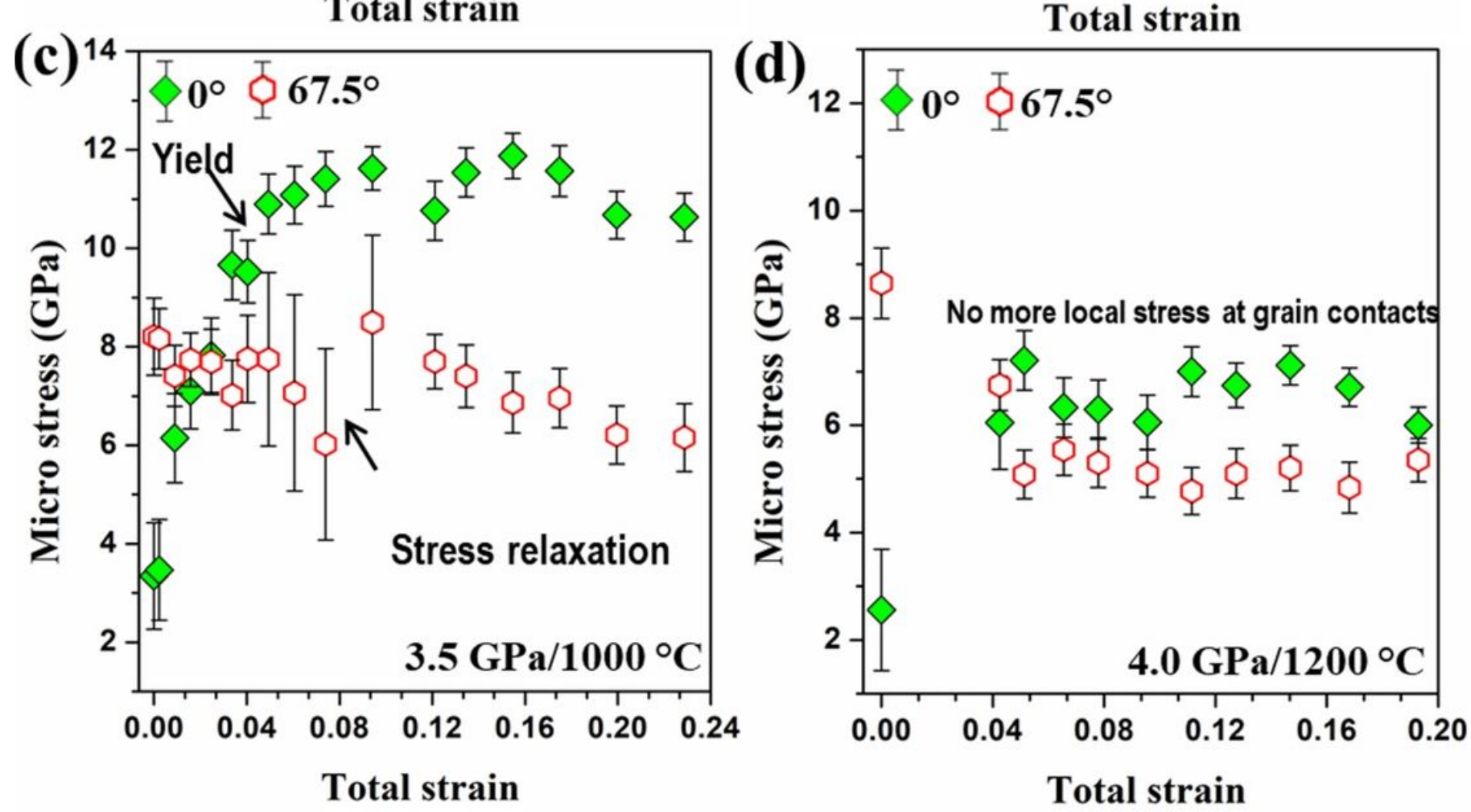

Figure 2

(Color online) Macro differential stress and micro stress of nc cBN as functions of total axial strain at different pressure and temperature conditions. (a) Representative stress strain curves of nc cBN deformed at $3.5 \mathrm{GPa} / \mathrm{RT}$ (green diamond), $3.5 \mathrm{GPa} / 1000{ }^{\circ} \mathrm{C}$ (hollow red hexagon) and $4.0 \mathrm{GPa} / 1200{ }^{\circ} \mathrm{C}$ (blue sphere); micro stresses determined from $0^{\circ}$ and $67.5^{\circ}$ azimuth angles for samples deformed at 3.5 $\mathrm{GPa} / \mathrm{RT}(\mathrm{b}), 3.5 \mathrm{GPa} / 1000{ }^{\circ} \mathrm{C}$ (c) and $4.0 \mathrm{GPa} / 1200^{\circ} \mathrm{C}$ (d). 


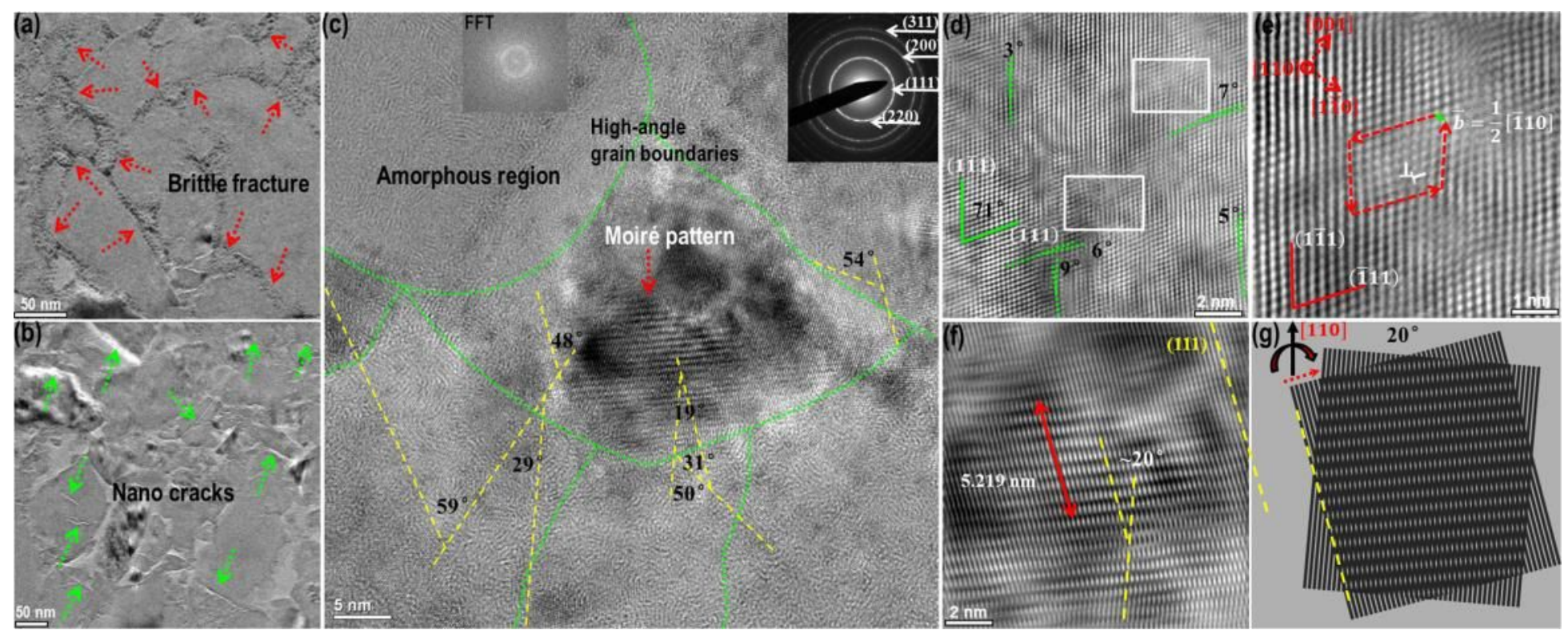

Figure 3

(Color online) TEM observations of nc cBN deformed at 3.5 GPa and RT. (a) Bright field TEM image; red dashed arrows pointing to the crushed particles between larger grains. (b) Bright field TEM image; green dashed arrows pointing to the propagating nanocracks. (c) HRTEM micrograph displaying various features in the deformed sample; see text for details. (d) IFFT image of low angle dislocations on lattice plane. White rectangular frames mark two Lomer Cottrell (L C) locks. (e) Enlarged image of the white rectangular frame in (d) showing the two extra half planes (stacking faults) in both the $\nabla 1$ and the $\nabla 1$ planes. (f) Enlarged Moiré fringes marked in (c). The twisted grain boundaries are due to grain rotation.

(g) Schematic drawing of twisted GB along axis in (f).
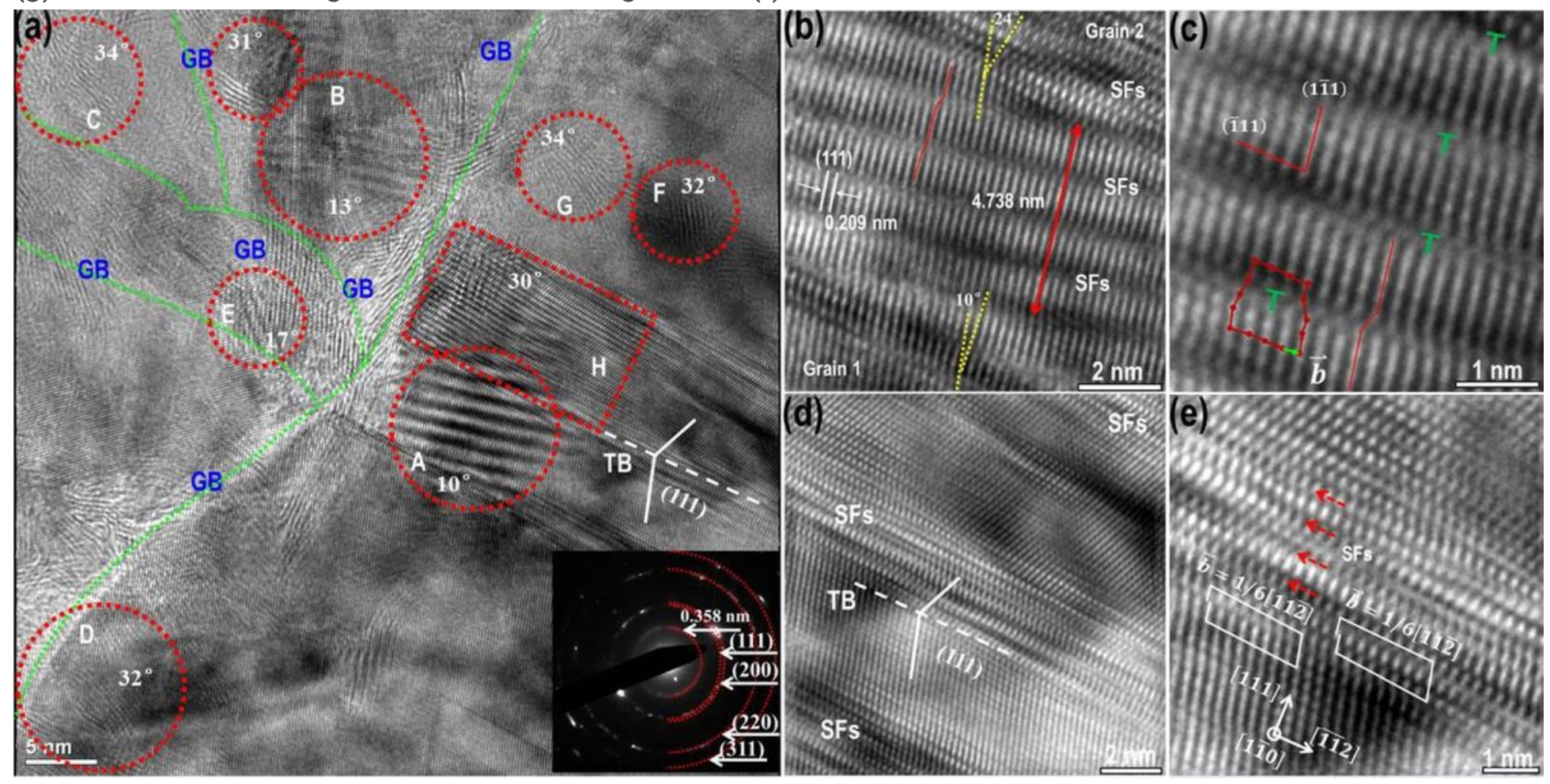


\section{Figure 4}

(Color online) HRTEM observations of dislocation movements in cBN sample deformed at $3.5 \mathrm{GPa}$ and $1000{ }^{\circ} \mathrm{C}$. (a) The red circles and rectangular fields (Moiré pattern) showing active grain rotation near high angle grain contacts. Twin boundary (stacking faults) along $\otimes 111 \rrbracket$ direction marked with white lines. The inset is a SAED of nc cBN with a superlattice diffraction ring . (b) Representative Moiré pattern IFFT image of the area " $A$ " in (a), indicating grain rotation induced stacking faults. (c) Enlarged Moiré image in (b). Burgers circuit enclosing a Shockley partial on $60^{\circ}$ full dislocation. Edge dislocations located at Moiré fringes are marked with " $\mathrm{T}$ ". (d) Representative IFFT image of twinning for the area " $\mathrm{H}$ " in (a), also showing grain rotation induced stacking faults. (e) Enlarged IFFT image of twinning structures of the area " $H$ " in (a); Burgers circuit enclosing two Shockley partials.
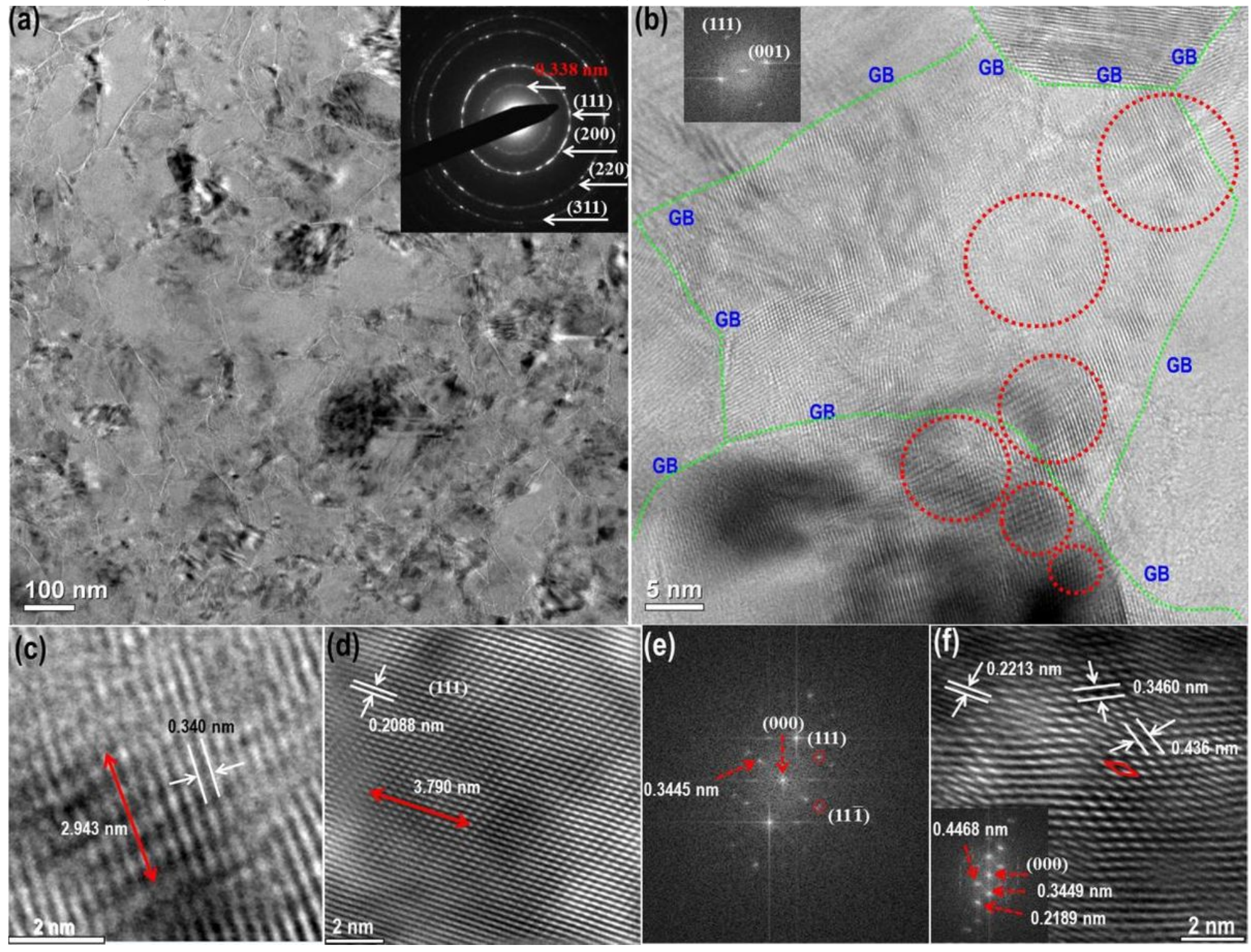

Figure 5

(Color online) HRTEM images of dislocation movements in cBN sample deformed at 4.0 GPa and 1200 ${ }^{\circ} \mathrm{C}$. (a) Bright field TEM image showing grain boundaries due to dynamic recrystallization of crushed grains under plastic flow. The inset is a SAED with an extra Debye ring of $0.338 \mathrm{~nm}$ in radius, indicating 
stress induced phase transition of $\mathrm{cBN}$ to hBN. (b) The red dashed circles represent active grain rotation induced dislocations near serrated grain boundaries. (c) Characteristic Moiré pattern IFFT image of the area enlarged from (b) on hBN. (d) Characteristic Moiré pattern IFFT image on cBN. (e) FFT taken from (c), which shows the superlattice plane with a d spacing of $0.3445 \mathrm{~nm}$ in hBN. (f) Typical superlattice structure on the sample. The rhombus represents the smallest repeating unit of the superstructure. Inset is the FFT taken from superlattice structure showing various superlattice planes.

\section{Supplementary Files}

This is a list of supplementary files associated with this preprint. Click to download.

- Supplementarylnformation.pdf 